\title{
Aerodynamic Simulation of Plunging Airfoil with Heat Effects and Lattice Boltzmann Technique
}

\author{
Hamed Saffarzadeh(1), Mohammad Hassan Djavareshkian ${ }^{(1, *)}$ \\ (1) Engineering Department, Ferdowsi university of Mashhad, Mashhad, IRAN \\ e-mail: hamed.saffarzadeh@gmail.com \\ (*) e-mail (corr. author): javareshkian@ferdowsi.ac.ir
}

\section{SUMMARY}

In this investigation, finite difference lattice Boltzmann method (FD_LBM) is developed to solve heat transfer effect behavior on the symmetrical and unsymmetrical airfoils with plunge oscillations. In this simulation, the equations of motion and energy are executed using LBM and FD simultaneously. The LB method is integrated with ghost flow for predicted curve boundary. The ghost flow method is a Cartesian-based method that, in addition to being practical and straightforward, retains many advantageous features of structured meshes, can be used for complex geometries, and has a high degree of flexibility. In other words, when the body oscillates, it is important to determine its position caused by the change in the mesh structure at any time. While the ghost method detects the object's position well, the new technique can capture the details of flow more accurately and stably than the other methods. Combining the ghost method with LBM provides a new technique that can investigate thermal behavior's effect on the airfoil with greater accuracy and stability. This combination of modern methods with high accuracy and stability in complex geometries has not been studied. The results are compared with the literature and show that this method has better convergence in different Reynolds and temperatures with changes at boundary conditions in the airfoil.

KEY WORDS: Lattice Boltzmann; ghost flow; computational fluid dynamics; plunge; heat transfer; total variation diminishing.

\section{INTRODUCTION}

Many types of research have been conducted to develop the field of Micro Aerial Vehicles (MAV). Recently, efforts have been made to improve aerodynamic performance at low Reynolds (LRN) flows. By utilizing the wings' oscillatory movement, many of these vehicles produce thrust and lift in the range of low Reynolds, even below 1000. MAV applications are widely and diversely used, from civilian and military programs to space exploration. In this range of Reynolds, the adverse pressure gradient plays a detrimental function in aerodynamic efficiency and thus induces flow separation of an airfoil. The operating conditions are different 
from ambient conditions. Therefore, various operational kinematics, wing section shapes, and Reynolds numbers have been investigated [1]. More research is needed to design and develop such configurations for LRN flow properties. The leading or trailing edge separation events increase drag and reduce lift [2]. In other recent studies [3], airfoils improved aerodynamic performance under surface heat treatment has been investigated. Due to the reduced size of the MAVs, compared to the older models, heat transfer effects are significant. Heat causes the boundary layer's relative growth in small wings with low Reynolds compared to larger airfoils. Succi [4] showed that heat transfer in a small area at the front edge affects significantly the airfoils performance. Their studies indicated different temperatures between the upper surface and the lower surface that had affected aerodynamic performance. Numerous studies have shown that biological flapping flyers utilize several unsteady mechanisms to keep afloat and control their flight [5]. However, these mechanisms role depends on wing deformation, wing kinematics, flight environment, and flyer size. Leading-edge vortex is generated aerodynamic force caused by a mechanism in flapping flight.

Lattice-Boltzmann method is a novel method for computational fluid dynamics (CFD) proven to be usable in a wide range of applications, such as turbulence flow [6], the multi-phase free surface [7], the flow of non-Newtonian, porous environment [8]. In the last decades, the LB method has been widely researched and used to solve various fluid problems. The inherent advantages of the LBM, as one of the approximate solutions of the Boltzmann equation with a lower computational cost, cause the significant development of this method for simulating flows. Boltzmann's basic equations, LBM, have the power to simulate continuous and rarefied flows [9], [10], so further details of the thermodynamic imbalance behavior can be examined in this way [11],[12]. It is often used instead of the Navier-Stokes equation (NSE) because the solution of the Boltzmann equation (BE) is much simpler [13]. An intrinsic feature of the LBM method is parallel computation and its application to complex geometries [14], which makes it more popular than traditional methods such as CFD methods.

In recent years, the combination of LBM with various CFD techniques has been studied, and different articles published. Matin et al. introduced a finite element-LB technique for binary fluids flow [15]. They stated that the FE-LBM scheme is a good solution and report satisfactory results of the accuracy of the experimental data obtained.

The purpose of this research is to develop a numerical method based on finite difference lattice Boltzmann (FD_LBM) to simulate the flow and heat transfer around an airfoil with plunge oscillations. The equations of motion and energy are solved using LBM and FD, respectively. The LB method is integrated with ghost flow for predicting the curve boundary. The ghost flow method is a Cartesian-based method that, in addition to being practical and straightforward, retains many of the advantageous features of structured meshes, can be used for complex geometries, and has a high degree of flexibility. The energy equation is solved by the FD method and total variation diminishing TVD technique. It is well known that when the diffusion fluxes (with dissipation behaviors) own negligible values regarding convective fluxes, almost all classical methods suffer serious instability errors. In order to resolve this issue, a novel TVD scheme is applied. The TVD characteristics of the following Hybrid LB method made it a valuable asset in solving these kinds of problems. In other words, the TVD scheme was applied to avoid numerical instability and improve the stability, precision, and convergence rate of the numerical solution. For this purpose, four limiters were used, and the results were compared in terms of accuracy and calculation time. Two symmetric and asymmetric airfoils, NACA0012 and SD7003 airfoils, in oscillation mode, have been investigated and the results were compared with those of previous articles. The accuracy of the results indicates this 
method's appropriacy for different geometries and different inlet flow conditions. The results also show this model's advantages compared to the Finite Volume (FV) model used in commercial software in terms of accuracy, speed of calculation, and CPU time.

\section{SOLUTION PROCEDURE}

\subsection{EQUATIONS OF LATTICE BOLTZMANN METHOD}

The D2Q9 lattice model is selected in this research. This lattice is denoted, for two dimensions and nine velocities vectors. The velocity coordinates are here expressed in lattice units. This method is applied to simulate the process of velocity in an incompressible flow. Here, a set of distribution functions is used to determine the flow field. The following evolution equations regulate this approach for the collision operator with a single-relaxation-time approximation. For a distribution function $f_{i}(\boldsymbol{x}, t)$, describing the probability of finding a fluid particle at the position $\boldsymbol{x}$ and time $t$ in Boltzmann equation reads as follows:

$$
f_{i}\left(\boldsymbol{x}+\Delta t_{s} \boldsymbol{c}_{i}, t+\Delta t_{s}\right)-f_{i}(\boldsymbol{x}, t)=\frac{\Delta t_{s}}{\tau_{s}}\left[f_{i}(\boldsymbol{x}, t)-f_{i}^{e q}(\boldsymbol{x}, t)\right]
$$

Where $\Delta t_{s}$ and $\tau_{s}$ are defined as the time step and relaxation time, respectively. The density distribution functions along the $i$-th direction are related to the normal node scheme $f$ and the corresponding equilibrium distribution functions $f_{i}^{e q}$. $i$ represents the discrete velocity direction $\boldsymbol{c}_{i}$. The equilibrium distribution function $f_{i}^{e q}(\boldsymbol{x}, t)$ is calculated as follows:

$$
f_{i}^{e q}(\boldsymbol{x}, t)=w_{i} \rho(\boldsymbol{x}, t)\left[1+\frac{\boldsymbol{c}_{i} \cdot \boldsymbol{u}(\boldsymbol{x}, t)}{C_{s}^{2}}+\frac{\left(\boldsymbol{c}_{i} \cdot \boldsymbol{u}(\boldsymbol{x}, t)\right)^{2}}{2 C_{s}^{4}}-\frac{(\boldsymbol{u}(\boldsymbol{x}, t))^{2}}{2 C_{s}^{2}}\right]
$$

Speed of sound in the lattice is $C_{s}$, in this model $C_{s} \cong c / \sqrt{3}$ the lattice velocity is $\boldsymbol{c}=\delta \boldsymbol{x} / \delta t$, where $\delta t$ and $\delta \boldsymbol{x}$ are time step and distance between two nodes respectively. The $\boldsymbol{e}_{i}$ parameters are units standardizing set of weights that denotes a discrete set of where velocity $i$ vary between 0 and 8 for the D2Q9 model that is obtained from Eq. (3). The macroscopic variables, namely fluid velocity and density in Eq. (2), are $\boldsymbol{u}$ and $\rho$ respectively.

$$
\boldsymbol{e}_{i}=\left\{\begin{array}{cc}
(0,0) & i=0 \\
\left(\cos \left[(i-1) \frac{\pi}{2}\right] \sin \left[(i-1) \frac{\pi}{2}\right] \cdot c\right) & i=1,2,3,4 \\
\left(\cos \left[(i-9) \frac{\pi}{4}\right] \sin \left[(i-9) \frac{\pi}{4}\right] \cdot \sqrt{2} c\right) & i=5,6,7,8
\end{array}\right.
$$

For an incompressible fluid flow, the kinematic viscosity $v$ is related to relaxation times by this correlation:

$$
v=\frac{1}{3}\left(\tau_{s}-0.5\right) C_{s}^{2} \Delta t_{s}
$$

Here in Eq. (4), the value of $\tau_{s}$ should be considered greater than 0.5 . The equilibrium distribution weighting factor for $i$-th direction is $w_{i}$ that is given as follows:

$$
w_{i}=\left\{\begin{array}{lr}
\frac{4}{9} & i=0 \\
\frac{1}{9} & i=1,3,5,7 \\
\frac{1}{36} & i=2,4,6,8
\end{array}\right.
$$

The macroscopic aerodynamic quantities such as pressure $P$, density $\rho$, and velocity $\boldsymbol{u}$ are obtained from the following relations: 


$$
\rho=\sum_{i=0}^{8} f_{i}, \quad \rho \boldsymbol{u}=\sum_{i=0}^{8} \boldsymbol{e}_{i} f_{i}, \quad P=\rho C_{s}^{2}
$$

The Boltzmann lattice approach used on the curve node grid is the style scheme of the cell vertex. The following equation applies to each node $r$ of the grid:

$$
f_{i}\left(r, t+\Delta t_{u}\right)=f_{i}(r, t)+\Delta t_{u} \sum_{k=0}^{k} S_{i k} f_{i}\left(r_{k}, t\right)-\frac{\Delta t_{u}}{\Delta t_{u}} \sum_{k=0}^{k} C_{i k}\left[f_{i}\left(r_{k}, t\right)-f_{i}^{e q}\left(r_{k}, t\right)\right]
$$

The derivation of Eq. (7) is provided in [16]. In this Eq. (7), $k=0$ denotes the pivotal node $r$. The summations run over the nodes $r_{k}$ connected to $r$; the quantities $S_{i k}$ and $C_{i k}$ represent the streaming, and collisional matrices of the $i$-th population related to the $k$-th node, respectively. The equilibrium distribution function is defined by Eq. (2). By solving Eqs. (1) and (7) simultaneously, the nodes' properties are obtained by intermediation at the overlapping points. The values of macroscopic variables and distribution functions for the surface of the solid boundary are obtained.

For the normal node and curve boundary in ghost fluid nodes, the following equations describe the post-collision and stream, respectively:

For collision:

$$
\tilde{f}_{i}^{N . e q}\left(\boldsymbol{x}_{i}, t\right)-\tilde{f}_{i}^{C . e q}\left(\boldsymbol{x}_{i}, t\right)=2 \tilde{f}_{i}^{n o n e q} \frac{\tau_{s}-\Delta t_{s}}{\tau_{s}-\frac{\Delta t_{s}}{2}}
$$

For stream:

$$
\tilde{f}_{i}^{C}-f_{i}^{N . e q}=\left[\left[1-\frac{\Delta t_{s}}{2 \cdot \tau_{s}}\right] \tilde{f}_{i}^{n o n e q}+\Delta t_{u} \sum_{k=0}^{k} S_{i k}\left[\grave{f}_{i k}^{e q}+\grave{f}_{i k}^{n o n e q}\right]\right] \frac{\Delta t_{u}}{\Delta t_{u}} \sum_{k=0}^{k} C_{i k} \dot{f}_{i k}^{n o n e q}
$$

Where $\tilde{f}_{i}^{C . e q}$ and $\tilde{f}_{i}^{\text {N.eq }}$ refer to the curve boundary equilibrium and normal node distribution function, and $\tilde{f}_{i}^{\text {noneq }}$ is the non-equilibrium distribution function. The quantities $\grave{f}_{i k}^{e q}$ and $\hat{f}_{i k}^{\text {noneq }}$ in Eq. (9) are defined as follows:

$$
\begin{gathered}
\grave{f}_{i k}^{e q}=f_{i k}^{N e q} \text { and } \hat{f}_{i k}^{N e q}=\left(1-\frac{\Delta t_{s}}{2 \tau_{s}}\right) \cdot f_{i k}^{N . n o n e q} \\
\text { or } \\
\grave{f}_{i k}^{e q}=f_{i k}^{C . e q} \text { and } \grave{f}_{i k}^{\text {noneq }}=f_{i k}^{\text {C.noneq }}
\end{gathered}
$$

The distribution functions $f_{i}^{\text {C.eq }}$ and $f_{i}^{\text {C.noneq }}$ of the right-hand side of Eq. (8) and $f_{i}^{\text {N.eq }}$ and $f_{i}^{\text {n.noneq }}$ of the right-hand side of Eq. (9) are evaluated by interpolation for the curve boundary procedure.

\subsection{BOUNDARY TREATMENT WITH THE GHOST FLUID METHOD}

In this part, the ghost fluid lattice Boltzmann method for detecting the solid boundary is simulated. The computational space is divided into two separate locales, a physical zone $\Omega_{p}$ and a solid zone $\Omega_{S}$. The fluid points $F P$ s are put inside the physical zone, while the ghost points GPs are situated inside the body points BPs contiguous to the fluid-solid boundary. 
Finding the Ghost points GP is the first step of this method. The ghost point must have a minimum of one mutual connection to the fluid nodes inner the solid domain. Then in the first step, the associated ghost point IPs must be identified. A point of image is thought to be on the typical line from the ghost point to the limit, so the purpose of the crossing point of the ordinary line with the limit boundary intersection point is at the midway (Figure 1). For interpolating the principal flow variables at the image, points use the information available from the neighboring fluid nodes after the $I P$ s is determined. In this case, the $I P$ neighbors are located in the fluid domain for the aerodynamic curve ranges, and the by-line insertion method is used to evaluate the estimation of the general variable state $\beta$ of the four nodes including $I P$, as shown by Eq. (11):

$$
\beta=a x+b y+c x y+d
$$

where $\beta$ denotes the value of general macroscopic variables, including density $\mathrm{r}$ and velocity $\boldsymbol{u}$ and temperature $T$. There are four unknown coefficients $a, b, c$, and $d$ in the two-line interpolation method. Therefore, four equations are needed to solve the unknowns. For this reason, the Cartesian IP coordinate values $(x, y)$ and $\boldsymbol{u}$ corresponding to the neighboring fluid points $N P$ are entered in Eq. (11). Once the coefficients are calculated, the $x$ and $y$ values of an $I P$ are inserted into Eq. (11) to get the general variable value $\boldsymbol{u}$. In this study, unlike the work of Tiwari and Vanke [17], these points were replaced with the correct tracking points, and in special cases, at least one of the NPs in the fluid field is not at one point in the image. This is done with the average between $G P$ and $I P$ points to predict the speed at a solid boundary. The four equations required to obtain the coefficients can be written in general terms as:

$$
\begin{gathered}
\alpha\left(\alpha_{i} x_{i}+\left(1-\alpha_{i}\right) \grave{x}_{l}\right)+b\left(\alpha_{i} y_{i}+\left(1-\alpha_{i}\right) \dot{y}_{l}\right)+ \\
+c\left(\alpha_{i} x_{i} y_{i}+\left(1-\alpha_{i}\right) \grave{y}_{l} \grave{x}_{l}\right)+d=\alpha_{i} \beta+\left(1-\alpha_{i}\right) \grave{\beta} \\
i=1,2,3,4
\end{gathered}
$$

$\alpha_{i}$ is calculated:

$$
\alpha_{i}= \begin{cases}0 & \text { if } N P_{t} \in \Omega_{\text {fluid }} \\ 1 & \text { if } N P_{t} \in \Omega_{\text {Solid }}\end{cases}
$$

The values of macroscopic parameters can be obtained by an image of any boundary conditions on the object with interpolation. Also, since the velocity of the boundary node is not known, the corresponding point of intersection as defined is changed by the normal gradient equation:

$$
\grave{\beta}=\frac{\partial \beta}{\partial \boldsymbol{n}}=a \boldsymbol{n}_{x}+b \boldsymbol{n}_{y}+c\left(x \boldsymbol{n}_{y}+y \boldsymbol{n}_{x}\right)
$$

where $\partial \beta / \partial \boldsymbol{n}$ is the gradient of the general macroscopic variable at the boundary intersection points of normal and the boundary and $\boldsymbol{n}$ is the normal unit vector toward the fluid domain.

Eq. (12) in a general form reads:

$$
\begin{gathered}
\alpha\left(\alpha_{i} x_{i}+\left(1-\alpha_{i}\right) \boldsymbol{n}_{x i}\right)+b\left(\alpha_{i} y_{i}+\left(1-\alpha_{i}\right) \boldsymbol{n}_{y i}\right)+ \\
+c\left(\alpha_{i} x_{i} y_{i}+\left(1-\alpha_{i}\right)\left(x_{i} \boldsymbol{n}_{x i}+y_{i} \boldsymbol{n}_{y i}\right)\right)+d=\alpha_{i} \beta+\left(1-\alpha_{i}\right)\left(\frac{\partial \beta}{\partial \boldsymbol{n}}\right)_{I B} \\
i=1,2,3,4
\end{gathered}
$$

For boundary conditions, the second-order extrapolation technique is applied. The central difference approximation formulation is used for the gradient of the general macroscopic variable at the boundary intersection points in the normal direction. 


$$
\text { Boundary condition : }\left\{\begin{array}{l}
\beta_{G P}=2 \beta_{B I}-\beta_{I P} \\
\left(\frac{\partial \beta}{\partial \boldsymbol{n}}\right)_{B I}=\frac{\beta_{I P}-\beta_{G P}}{\Delta \varepsilon}
\end{array}\right.
$$

$\Delta \varepsilon$ represents the spatial distance between the corresponding GP and IP. Thus, one has $(\partial \rho / \partial \mathbf{n})_{B I}=0$ mean density of ghost points equal to the density of image point from Eq. (16) [18]:

$$
\rho_{G P}=\rho_{I P}
$$

In this stage, knowing the value of the macroscopic variables by $f_{i}^{e q}$ in Eq. (2) the ghost point and $\rho$ ghost can be calculated. No equilibrium distribution functions $f_{i}^{\text {noneq }}$ or similar procedure are extrapolated to a density calculation. Tiwari [17] noted that this method is accurate for extrapolating the first-order non-equilibrium parts whereas extrapolating the no equilibrium part becomes second order. By having equilibrium and non-equilibrium of internal energy distribution and density functions, distribution ghost points can be obtained.

$$
\tilde{f}_{I G P}=f_{i G P}^{e q}+f_{i G P}^{n o n e q}
$$

\subsection{THE LB METHOD WITH TOTAL VARIATION DIMINISHING}

In this scheme, the hybrid finite-difference is used to solve the energy equation. The function of equilibrium distribution described the momentum relaxation time, Lattice Boltzmann equation, Eqs. (1-10). The key alteration here is the extension of energy equation simulation:

$$
\frac{\partial \theta}{\partial \tau}+\frac{\partial(\boldsymbol{U} \theta)}{\partial x}+\frac{\partial(\boldsymbol{U} \theta)}{\partial y}=\nabla^{2} \theta
$$

Hence, a TVD scheme is used for the definition of convective terms of $\theta$ and $\boldsymbol{U}$ is strem velocity. According to Guo and Shu [19], higher stability can be achieved for an extra conservation equation with an additional distribution function. Also, the hybrid method's weakness is numerical instability which is not particularly suitable for low Reynolds.

However, this study shows that, contrary to popular belief, existing TVD schemes not only do not suffer from this problem, but they can even improve the accuracy, stability, and convergence rate of numerical solutions. As mentioned in the present study, the hybrid finite difference is used to solve the energy equation.

The key discrete temperature field equation reads as follows:

$$
\frac{\theta_{r}^{\text {new }}-\theta_{r}}{\partial \tau}+\frac{(\boldsymbol{u} \theta)_{e}-(\boldsymbol{u} \theta)_{w}}{\partial x}+\frac{(\boldsymbol{U} \theta)_{n}-(\boldsymbol{U} \theta)_{s}}{\partial y}=\left(\frac{\theta_{E}-2 \theta_{r}+\theta_{W}}{\Delta x^{2}}+\frac{\theta_{N}-2 \theta_{r}+\theta_{S}}{\Delta y^{2}}\right)
$$

To solve the above equation with a relatively low dissipation, a TVD scheme is applied to the convective values of $\theta$ (i.e., $\theta_{e}, \theta_{w}, \ldots$ ). FD and FV methods have been considered to be accurate numerical methods with high-resolution techniques over the past few decades. Hirsch [21] presented a more detailed study of the new TVD limiting functions $\psi=\psi(l)$, with $l$ being $l=\left(\theta_{r}-\theta_{w} / \theta_{e}-\theta_{r}\right)$, to calculate the convective terms in the flows with severe parameter gradients. $\theta_{e}$, for example, can be obtained from the following relation:

$$
\theta_{e}=\theta_{r}+\frac{1}{2} \psi(l)\left(\theta_{E}-\theta_{r}\right)
$$

$\psi(l)$ can change any limiter or generate regular estimates for convection fluxes. These schemes have certain stability and accuracy [22].

The Superbee limiter function is very common not only because of its TVD characteristics but also because of its fine precision. It is considered as one of the best limiter functions available. Minmod has better stability and faster convergence than Superbee but has less accuracy due to 
additional dissipations. Roe [23] proposed these two limiter functions. Therefore, these limiters are used for the measurement of convective fluxes.

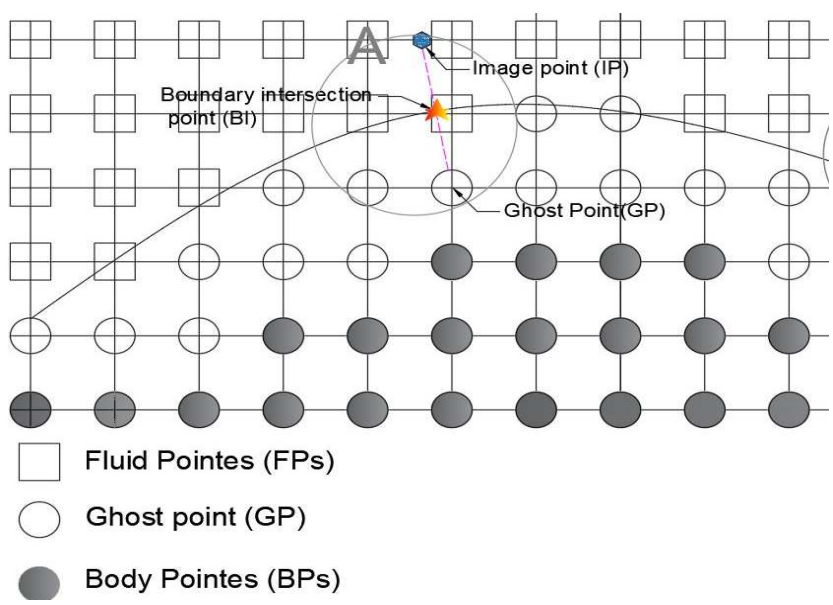

Fig. 1 Schematic illustration of the immersed boundary treatment

$$
\begin{gathered}
\psi_{\text {Superbee }}=\max [0, \min (2 l, 1), \min (l, 2)] \\
\psi_{\text {Minmod }}=\left\{\begin{array}{lll}
\min (l, 1) & \text { if } & l \geq 0 \\
0 & \text { if } & l \leq 0
\end{array}\right. \\
\psi_{\text {Van Albada }}=\frac{2}{1+l^{2}} \\
\psi_{\text {Van Leer }}=\frac{2}{1+l}
\end{gathered}
$$

\section{RESULTS AND DISCUSSION}

\subsection{PROBLEM STATEMENT}

In this study, the effect of heat on an airfoil at Plunge oscillatory motion is investigated by the novel hybrid numerical method that consists of finite FD_LBM combine with ghost flow. This airfoil is one of the most widely used airfoils for numerical method validation. The simulation is computed at various Reynolds numbers, plunging NACA 0012 airfoil with a reduced frequency of 3.93, and no dimensional plunging amplitude of 0.0125 . The mesh size depends on the length of the airfoil chord and domain size. Different mesh sizes are selected in a square domain with the length $L$ of the lattice's chord 100 units. The obtained drag coefficients for different mesh sizes are compared to each other and to the experimental data.

The effect of mesh size is shown in Table 1, for $R e=50,000$ and $R e=100,000$. As depicted in Table 1 , it can be seen that as the size of the mesh increased, the difference between the results of the simulation decreased. For choosing the best size mesh, the result has been compared with experimental data in Ref [20]. In Table 1, when the mesh size is increased from $1000 \times 1000$ to $6800 \times 1500$, the maximum relative error of $0.1 \%$ is seen in the drag coefficient. Thus, all the following sections' results are obtained using a grid size of $4500 \times 1500$ in a rectangular domain. Thus, the drag coefficient variation $C d$ with different airfoil conditions was estimated. 
Table 1 Mesh size effects on the results of drag coefficient at Reynolds $0.5 \times 10^{5}$ and $10^{5}$

\begin{tabular}{|c|c|c|c|c|}
\hline$R e$ & Size & Cd simulation & Cd in NASA Report [20] & Error percentage \\
\hline \multirow[t]{5}{*}{$R e=50,000$} & $1000 \times 1000$ & 0.08424 & \multirow{5}{*}{0.052} & $-62.0 \%$ \\
\hline & $2000 \times 1500$ & 0.07074 & & $-36.0 \%$ \\
\hline & $3000 \times 1500$ & 0.051064 & & $1.8 \%$ \\
\hline & $4500 \times 1500$ & 0.0511054 & & $1.7 \%$ \\
\hline & $6800 \times 1500$ & 0.051136 & & $1.7 \%$ \\
\hline \multirow[t]{5}{*}{$R e=100,000$} & $1000 \times 1000$ & 0.06306 & \multirow{5}{*}{0.037} & $-70.5 \%$ \\
\hline & $2000 \times 1500$ & 0.05201 & & $-40.6 \%$ \\
\hline & $3000 \times 1500$ & 0.03639 & & $1.6 \%$ \\
\hline & $4500 \times 1500$ & 0.03643 & & $1.5 \%$ \\
\hline & $6800 \times 1500$ & 0.03647 & & $1.4 \%$ \\
\hline
\end{tabular}

The following relation expresses the harmonic motion of the immersion in the vertical direction:

$$
Y(\zeta)=\frac{A}{C h o} \sin (2 k \zeta)
$$

$Y(\zeta)$ is the dimensionless airfoil amplitude, $A$ is amplitudes frequency and Cho is airfoil chord also $\zeta=\frac{t U_{\infty}}{C h o}$ and $k_{f}=\pi \cdot$ freq $\cdot \frac{C h o}{U_{\infty}}$ are the dimensionless time and reduced frequency respectively. For validation of the new LB method for plunge motion, the simulation results are compared with Lian and Shyy [24] who studied the aerodynamics coefficient at $A / C h o=0.0125$ and $R e=20,000$ with 2D and FV in viscous -laminar model and meshing above 36500 nodes. The drag coefficients at $k_{f}=3.93$ are computed.

As shown in Figures 2 and 3, there is good overlap in the time history of the plunge drag between present calculations and the results of Lian and Shyy [24]. The difference between the results of the $C d$ and lift coefficient $C l$ is less than one percent. This comparison shows the accuracy of this simulation.

It indicates the use of a suitable mesh with almost orthogonal cells and determining appropriate parameters in the simulation. Sutherland and Eucken's relations are used to calculate the thermal conductivity and dynamic viscosity [25], [26]. Dynamic viscosity increases with the increase in temperature. As a result, the drag coefficient increases with the increase of the dynamic viscosity. For Newtonian fluids, the viscosity shear stress is proportional to the dynamic viscosity and the wall's normal velocity slope. In aerodynamics, drag is often undesirable and is known as a resistive force. The effect of temperature on the drag in the range of $200 \mathrm{~K}$ to $400 \mathrm{~K}$ is shown in Figure 4. As can be seen, the rise of the temperature from $200 \mathrm{~K}$ to $300 \mathrm{~K}$ increases the drag coefficient by $24.6 \%$, from $300 \mathrm{~K}$ to $400 \mathrm{~K}$. This indicates a more significant effect of dynamic viscosity at low temperatures, which affects the amount of drag. 


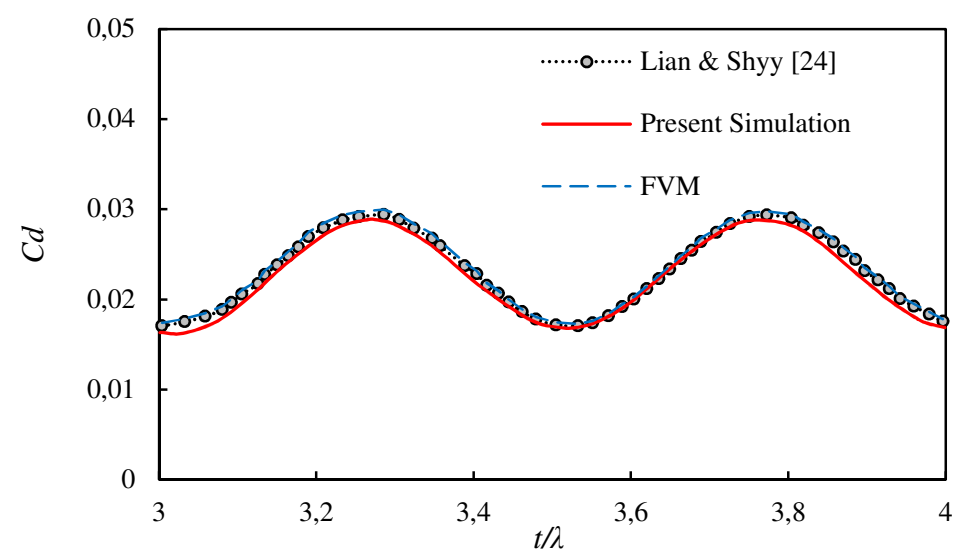

Fig. 2 Drag coefficient on Plunging NACA0012 airfoil at $R e=20,000, A / C h o=0.0125, k_{f}=3.93$

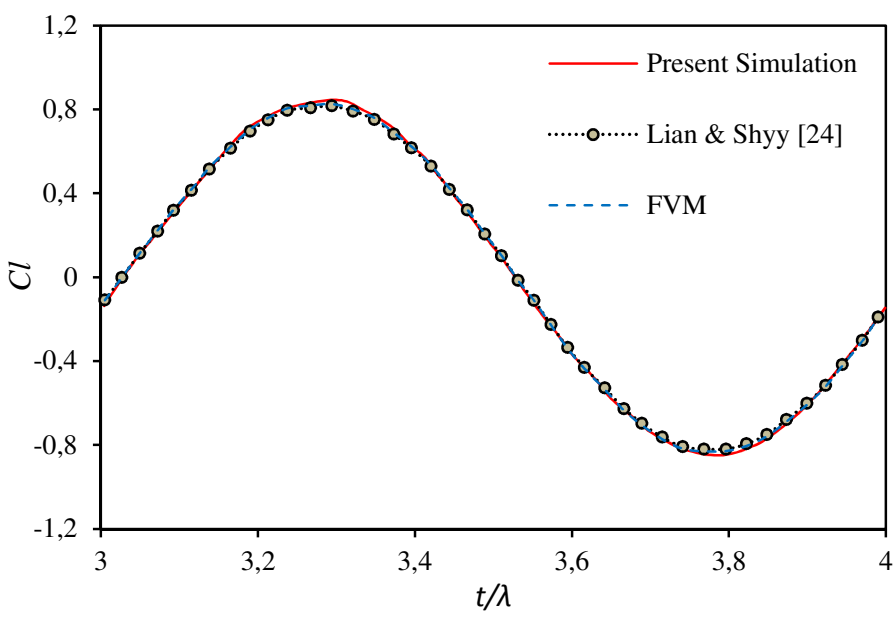

Fig. 3 Lift coefficient on Plunging NACA0012 airfoil at Re $=20,000, A / C h o=0.0125, k_{f}=3.93$

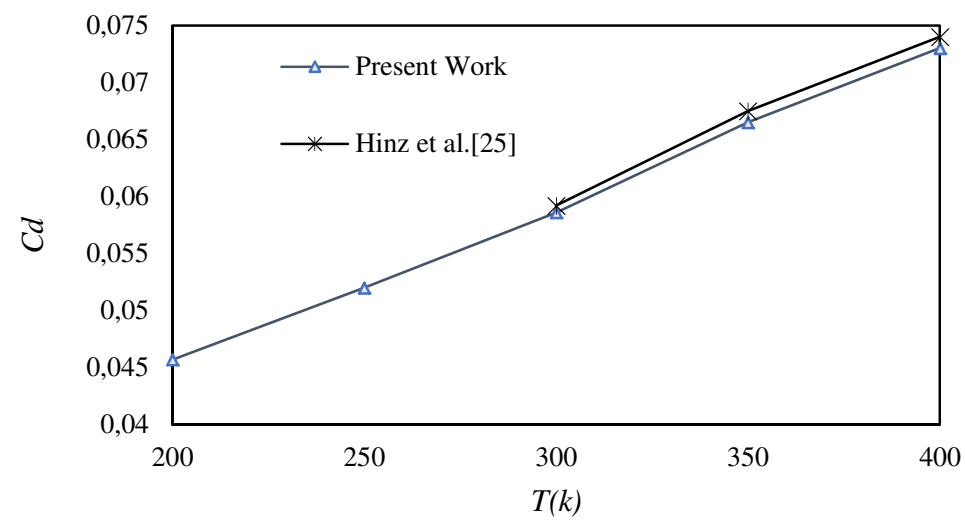

Fig. 4 Comparison of NACA0012 drag coefficient result with Hinz et al. [25] at Re=3000

\subsection{RESULTS}

It should be noted that, since the solver initiation in this simulation is static, the results for the initial periods for the aerodynamic coefficients do not reach their quasi-steady state, so that 
the data after a few intervals have to be accepted. To compare the results with the FV method, results reported by Hinz et al. [25] are considered. The results of airfoil plunge oscillatory motion with Reynolds number 20,000, Mach number 0.001, reduce frequency 3.93, dimensional oscillation amplitude 0.0125 are shown in Figure 5. In this study, heat transfer in heating and cooling conditions on plunge oscillating airfoil is investigated. For this purpose, the NACA 0012 airfoil was simulated at streamflow temperatures of $300 \mathrm{~K}$ and surface airfoil temperatures of $200 \mathrm{~K}$ to $400 \mathrm{~K}$. Figure 5 shows the instantaneous lift coefficients for the oscillation period at temperatures of $200 \mathrm{~K}, 300 \mathrm{~K}$, and $400 \mathrm{~K}$. As can be seen, with increasing cooling, the instantaneous lifting coefficient and heating reduce the maximum lifting.

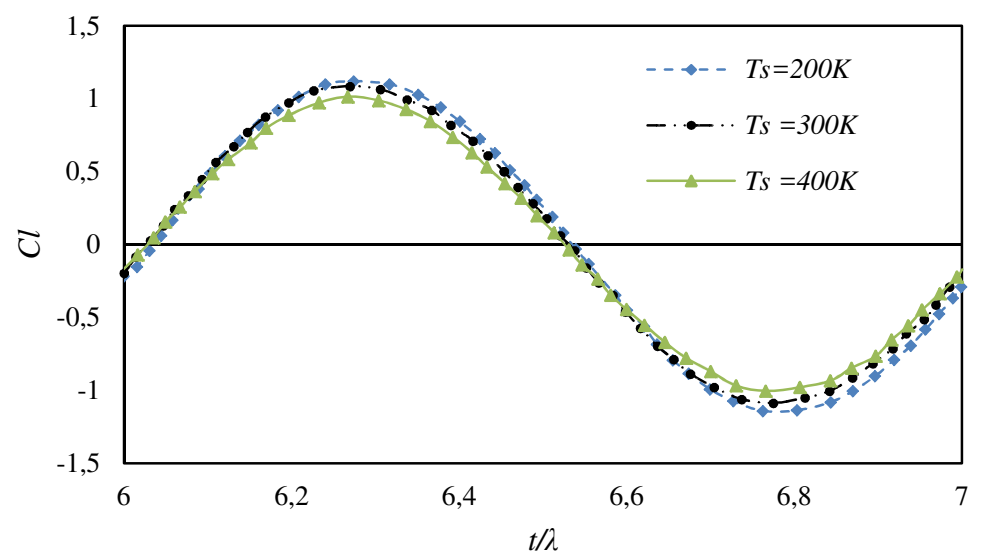

Fig. 5 Lift coefficient at different surface temperatures for NACA0012 at Reynolds 20,000 reduces the frequency by 3.93 and dimensionless oscillation amplitude by 0.0125

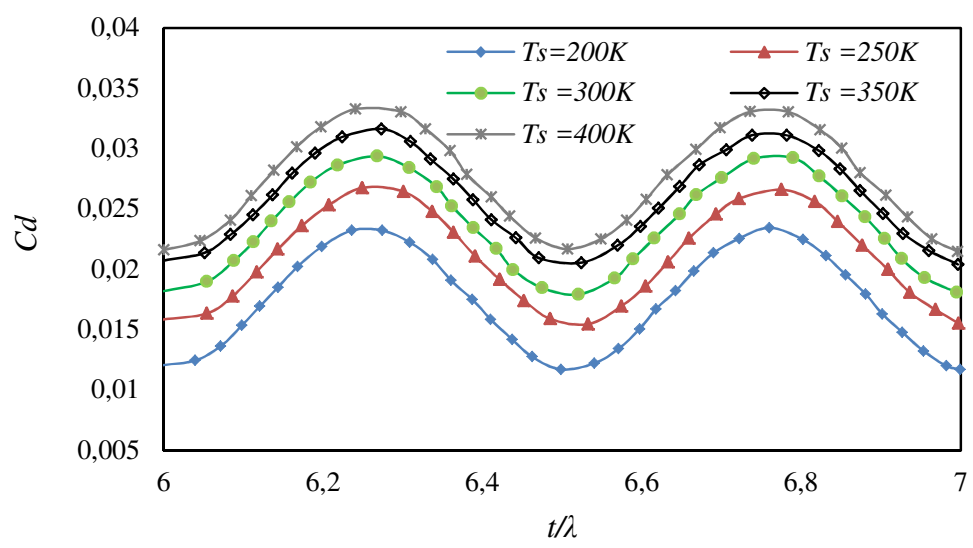

Fig. 6 Drag coefficient at different surface temperature for NACA0012 at Reynolds 20,000 for reduced frequency by 3.93 and dimensionless oscillation amplitude by 0.0125

Table 2 shows the maximum lift coefficients at different temperatures. One can notice that the lift coefficient curve's sinusoidal shape has slightly changed due to cooling and heating.

Table 2 Maximum lift coefficients at different airfoil surface temperatures

\begin{tabular}{|l|l|l|l|l|l|}
\hline Ts & $400 K$ & $350 K$ & $300 K$ & $250 K$ & $200 K$ \\
\hline$C l_{\max }$ & 1.0115 & 1.0574 & 1.0901 & 1.1191 & 1.1293 \\
\hline
\end{tabular}




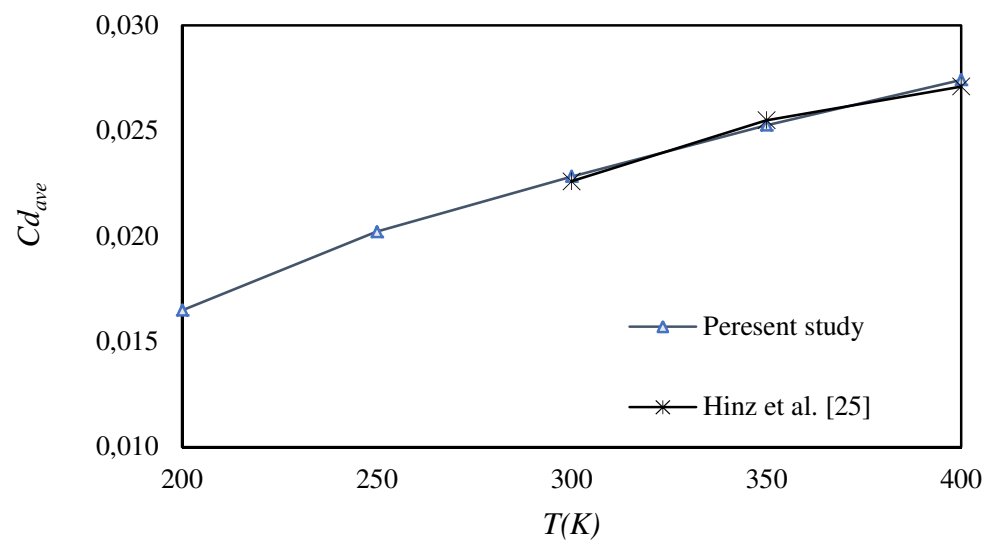

Fig. 7 Average Cd at different surface temperatures for NACA 0012 at Reynolds 20,000 reduces the frequency by 3.93 and dimensionless oscillation amplitude by 0.0125

Figure 6 shows the instantaneous drag coefficients. The efficacy of heat transfer on the drag coefficient is very similar to lift coefficients and increases with the increase of the temperature and decreases with the decrease of the temperature. Figure 7 shows that the oscillatory average drag coefficient is affected by heat transfer. Also, with an increase of 50 degrees of the airfoil surface temperature relative to the free flow, the average drag coefficient of $10.7 \%$ can be seen. With an increase of 100 degrees, the average drag coefficient is approximately a $20.2 \%$ increase.

In comparison, the mean drag coefficient decreased by $12.98 \%$ with the cooling of 50 degrees and with the 100 -degree it decreased by $38.91 \%$. Figures of the drag coefficient show that cooling has a greater effect on the drag coefficient than on heating. The increasing and decreasing trend of the oscillation coefficient relative to the oscillatory state may be due to dynamic viscosity changes, which are easily determined in the LB model with Eq. (6) at any particular time. Moreover, heat changes can be measured using the hybrid FD method with LB. The rise in viscous drag is related to the increase in surface temperature and the change in the viscous boundary layer's fluid properties. The Lattice method and the ghost boundary curve model represent variations in fluid viscosity by correctly approximating the airfoil's boundary layer. The cooling state's viscosity variations are greater than the heating state [27], so the influence of the cooling state's coefficient changes may be greater than the heating effect. Figures of the instantaneous and average drag coefficients also indicate that by raising the surface temperature, the airflow decreases the heat transfer over the instantaneous and intermediate drag coefficients due to the boundary layer's unsteady heating status. Oscillating airfoil has stable and more appropriate results during the boundary layer's cooling mode while heating the airfoil's surface reduces the flow time and increases the instability. This instability in conventional methods can affect the simulation algorithm and lead to divergence in results, but in this simulation using the TVD method, the stability and convergence rate have also increased. Table 3 shows that simulations without TVD at $400 \mathrm{~K}$ has been divergence. Another conclusion drawn from the influence of heat transfer on the oscillation drag coefficient is that if the airfoil produces zero drag or no thrust at a specified frequency and amplitude, it can be converted into a thrustgeneration state. To illustrate this, we increased the frequency of the deceleration compared to the previous simulation. Figures 8 and 9 show the instantaneous and average drag coefficients, respectively. Figure 8 shown the coefficient of drag at different surface temperatures for an oscillating cycle. As can be seen in Figure 9 for the non-heat transfer mode (airfoil temperature and streamflow $300 \mathrm{~K}$ ), the average drag coefficient is approximately zero, and the temperature rise increases the drag and the decrease of the temperature causes the negative drag or thrust. 
Figures 10 show when the surface temperature increases, the coefficient of lift increases. This amount of change is less compared to the drag coefficient changes.

Table 3 Comparison of the drag coefficient for TVD code with Superbee, Minmod limiter, and without TVD for Plunging NACA0012 at Reynolds 2000 for reduced frequency by 3.93 and dimensionless oscillation amplitude by 0.0125

\begin{tabular}{|c|c|c|c|c|c|c|c|}
\hline Method & Grid Size & $\begin{array}{l}\text { Cd dave at } \\
T=200 \mathrm{~K}\end{array}$ & $\begin{array}{l}\text { Cd dave at } \\
T=300 K\end{array}$ & CPU time & $\begin{array}{c}C d_{\text {ave }} a t \\
T=300 K \\
{[21]}\end{array}$ & $\begin{array}{l}\text { Cd ave at } \\
T=400 K\end{array}$ & $\begin{array}{c}C d_{\text {ave }} a t \\
T=400 K \\
{[21]}\end{array}$ \\
\hline \multirow{2}{*}{$L B M$} & $4500 \times 1500$ & 0.016981 & 0.022144 & 243700 & \multirow{10}{*}{0.022600} & $\mathrm{NaN}^{*}$ & \multirow{10}{*}{0.027100} \\
\hline & $6800 \times 1500$ & 0.015992 & 0.022506 & 372620 & & $N a N$ & \\
\hline \multirow{2}{*}{ Minmod } & $4500 \times 1500$ & 0.016322 & 0.222582 & 226640 & & $N a N$ & \\
\hline & $6800 \times 1500$ & 0.016319 & 0.022486 & 338380 & & 0.026600 & \\
\hline \multirow{2}{*}{ Superbee } & $4500 \times 1500$ & 0.016487 & 0.022829 & 237490 & & 0.027420 & \\
\hline & $6800 \times 1500$ & 0.016454 & 0.022600 & 353080 & & 0.027145 & \\
\hline \multirow{2}{*}{ Van Albada } & $4500 \times 1500$ & 0.015283 & 0.019930 & 220760 & & $\mathrm{NaN}$ & \\
\hline & $6800 \times 1500$ & 0.015192 & 0.021381 & 328290 & & 0.026016 & \\
\hline \multirow{2}{*}{ Van Leer } & $4500 \times 1500$ & 0.015454 & 0.021463 & 217790 & & $N a N$ & \\
\hline & $6800 \times 1500$ & 0.016792 & 0.023631 & 317710 & & 0.027642 & \\
\hline
\end{tabular}

* Not a Number

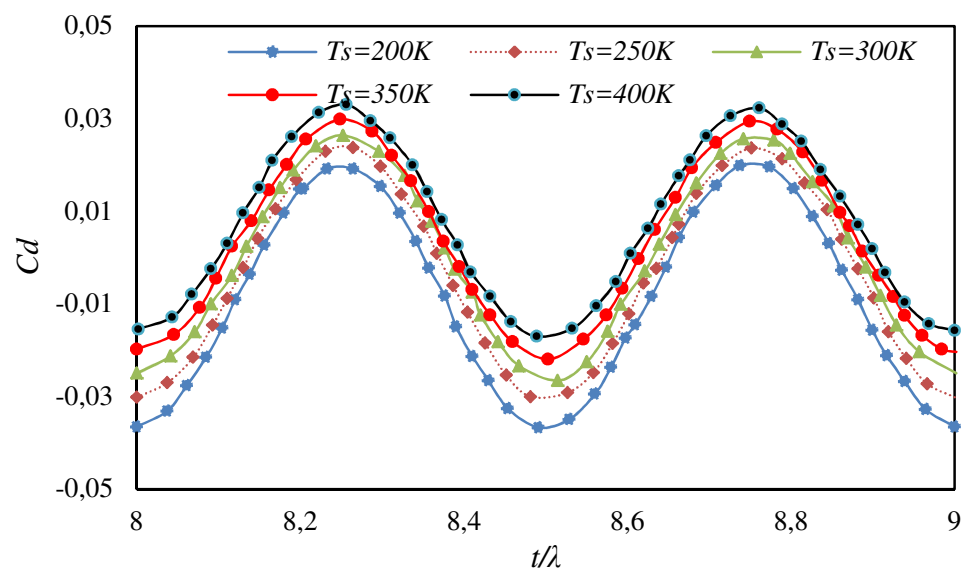

Fig. 8 Drag coefficient at different surface temperatures for NACA0012 at Reynolds 20,000 reduces the frequency by 7.86 and dimensionless oscillation amplitude by 0.0125

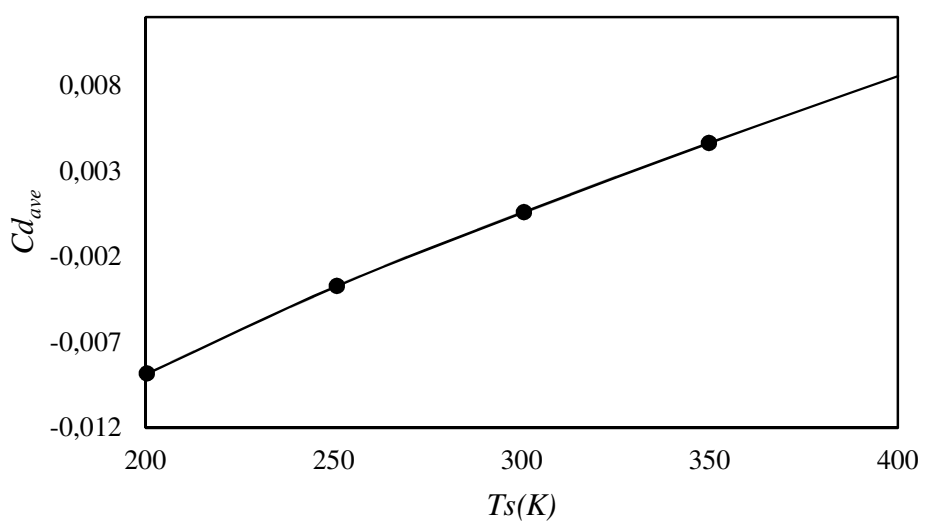

Fig. 9 The average drag coefficient at different surface temperatures for NACA0012 at Reynolds 20,000 reduces the frequency by 7.86 and dimensionless oscillation amplitude by 0.0125 


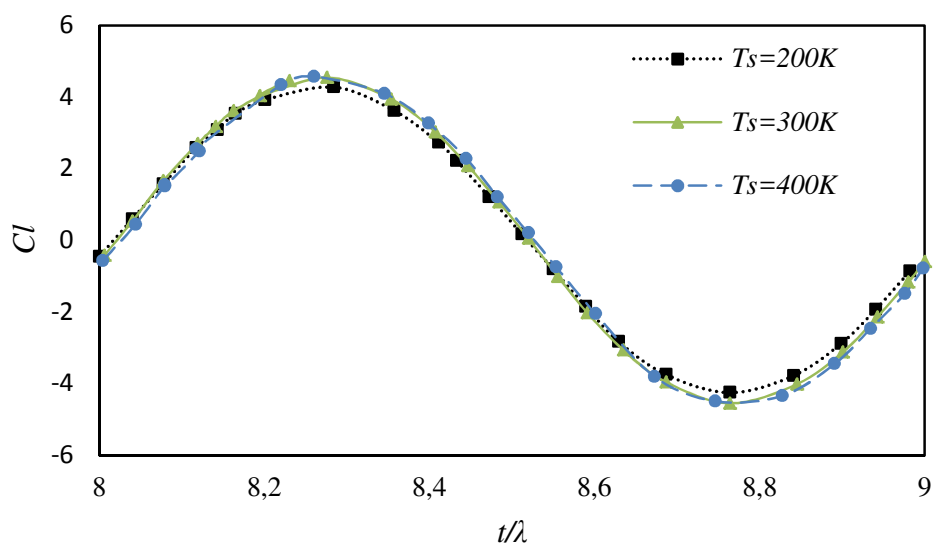

Fig. 10 Lift coefficient at different surface temperatures for NACA0012 at Reynolds 20,000 reducing the frequency by 7.86 and dimensionless oscillation amplitude by 0.0125

The change in oscillation frequency attributed to the approach suggested in this simulation does not affect the convergence and is stable. As seen at higher frequencies, the effect of heat transfer is attributed to less heat transfer time. In other terms, frequency $k_{f}=3.93$ for the maximum drag from $200 \mathrm{~K}$ to $400 \mathrm{~K}$ is reduced for almost $10.5 \%$ while frequency $k_{f}=7.86$ for about $6.5 \%$.

One of the most commonly used airfoils for MAV is the Selig-Donovan airfoil, SD7003, which often explores heat transfer's effect using the above technique. The following Figures 11 and 12 indicate that cooling mode positively affects the airfoil's aerodynamic performance, i.e. the lift coefficient will increase and the drag coefficient will decrease. However, the heating of the airfoil surface by decreasing the lift coefficient and increasing the drag coefficient negatively affects the airfoil performance. So, the exact behavior of the previous airfoil can be observed here.

Table 4 shows the average lift of drag ratio in heating, cooling, and without heat transfer on the airfoil surface. Comparing the non-heat-transfer state with cooling, the lift-to-drag average ratio increased by 68.54 percent and decreased by 19.46 percent in heating, which is a positive effect of cooling on this airfoil type.

At the end of this study, we will discuss that due to temperature changes in the airfoil surface and dynamic solutions, improvements should be made by combining the LB_FD process with the TVD limiter.

Table 4 Lift and drag average coefficients for different airfoil surface temperatures

\begin{tabular}{|llll|}
\hline Airfoil temperature & Cd ave & Clave & Clave $_{\text {avd }}$ \\
\hline$T s=200 \mathrm{~K}$ & 0.0157 & 0.306 & 19.4904 \\
\hline$T s=300 \mathrm{~K}$ & 0.022 & 0.235 & 10.6818 \\
\hline$T s=400 \mathrm{~K}$ & 0.027 & 0.185 & 6.8518 \\
\hline
\end{tabular}




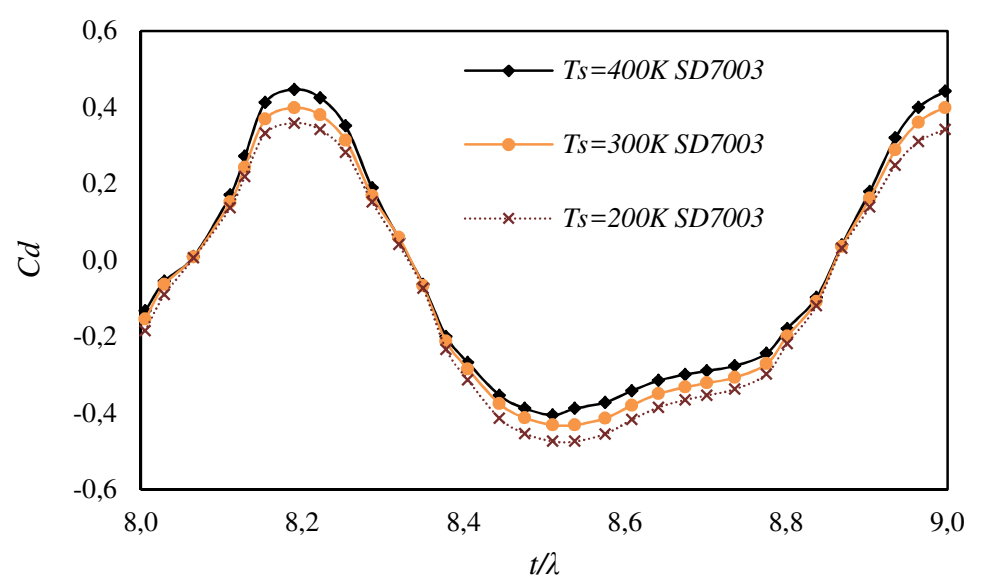

Fig. 11 Drag coefficient at different surface temperatures for SD7003 at Reynolds 20,000, reduces the frequency by 3.93 and dimensionless oscillation amplitude by 0.0125

To increase computational speed, different limiters; Superbee, Minmod, Van Leer, and Van Albada, with a TVD scheme, are used in this simulation. The results of Superbee limiters proved it to be more adequate than Minmod limiters. When the temperature rises to near $400 K$, the simulation in less grid for Van Albada and Van Leer limiters fails to simulate plunging airfoil.

The best limiter to achieve greater stability is the Superbee. As described in [19], the approved Superbee limiter produces a significant numerical dispersion, limiting the major impact on numerical models' accuracy. The appropriate limiter should be selected based on the consistency and accuracy of the actual results. As shown in Table 4, the above method converges to a wider temperature range, and all limiters obtain acceptable results. Simply, the Superbee limiter obtains higher accuracy than the Minmod. TVD schemes are supposed to require more resolution time than LB-FD code due to additional computation. However, an exemplary comparison reveals that the current hybrid codes are more precise and require less CPU time than the LBM code.

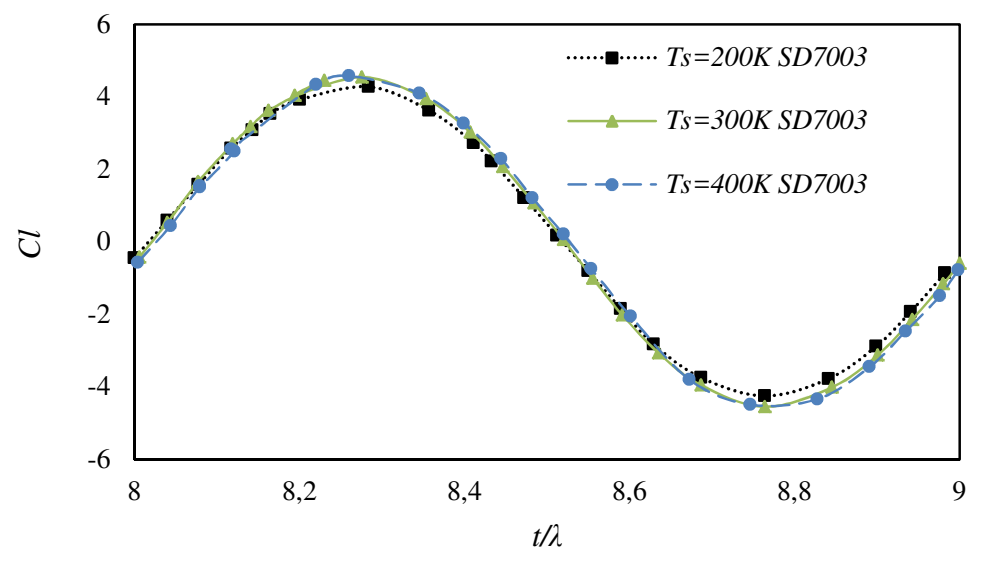

Fig. 12 Lift coefficient at different surface temperatures for SD7003 at Reynolds 20,000, reduces the frequency by 3.93 and dimensionless oscillation amplitude by 0.0125 


\section{CONCLUSIONS}

In the present research, combination of the finite deference and lattice Boltzmann numerical method with TVD characteristics is proposed to solve heat transfer behavior on the symmetrical and unsymmetrical airfoil, NACA0012 and SD7003, with plunge oscillations and to simulate airfoil surface boundary with ghost flow method. Boltzmann equations increase the resolution rate to calculate the dynamic behavior of the oscillation airfoil. Its combination with FD is used to calculate the heat transfer behavior and to converge the results over a wider range of the TVD method used as well as the Lattice coefficients. Also, since the LBM network is regular and Cartesian coordinates, the ghost flow method is used to obtain higher accuracy for identifying solid boundary curves.

The main findings of this research are as follows:

- The combination of the finite-difference and lattice Boltzmann method with the TVD characteristics is performed to obtain aerodynamic parameters, the energy equation is solved with FD and TVD technique, and with scalar equation solved with FD a flux limiter is derived.

- It is combined in which fluid flow parameters are obtained in LBM, and the scalar equation is solved with the FD method with various flux limiter functions.

- The new method can capture the details of flow more accurately and stably than other methods, at least in low- Reynolds-number flow.

- The well-known functions of Superbee, Minmod, Van Albada and Van Leer were selected as flux limiters to be implemented in this study of benchmarks, and excellent compatibility with findings within available literature was obtained.

- The innovative numerical method also has a lower computational cost than similar methods.

- The hybrid method is also numerically much more efficient than the LBM code laminar flow.

- The innovative numerical technique is also estimated based on its computational costs. The hybrid method has been shown to be much more numerically effective than the LBM code for laminar flow as opposed to popular assumption.

- Eventually, one of the most important results of this new combined method lies in the convergence rate of the solution. For simulated flow on airfoils, TVD models provide converging faster and easier, resulting in CPU shorter computation time and lower computational costs.

\section{NOMENCLATURE}

\begin{tabular}{|c|l|c|l|}
\hline$a, b, c, d$ & Coefficients of equation & $f_{i}(x, t)$ & Distribution function \\
\hline$A$ & Amplitudes oscillation & $f_{i}^{e q}$ & Equilibrium distribution functions \\
\hline$A / C h o$ & $\begin{array}{l}\text { Non-dimensional amplitudes frequency } \\
\text { to chord }\end{array}$ & $\tilde{f}_{i}^{\text {C.eq }}$ & The curve boundary equilibrium \\
\hline$C$ & Lattice velocity & $\tilde{f}_{i}^{\text {N.eq }}$ & The normal node distribution function \\
\hline$C d$ & Drag coefficient & $\tilde{f}_{i}^{\text {noneq }}$ & Non-equilibrium distribution function \\
\hline$C d_{\text {ave }}$ & Drag average coefficients & $k_{f}$ & Reduced frequency \\
\hline Cho & Chord of the airfoil & $N u$ & Mean Nusselt number on the airfoil \\
\hline$C l$ & Lift coefficient & $P r$ & Prandtl Number \\
\hline$C l_{\text {ave }}$ & Lift average coefficients & $P$ & Pressure \\
\hline$C_{P}$ & Specific heat & $P^{*}$ & Non-dimensional pressure \\
\hline
\end{tabular}




\begin{tabular}{|c|c|c|c|}
\hline$C_{s}$ & Speed of sound in the lattice & $R e$ & Reynolds Number \\
\hline $\boldsymbol{e}_{i}$ & Discrete velocity & $T$ & Temperature \\
\hline freq & Frequency & $T s$ & Different surface temperature \\
\hline$M$ & Mach number & \multirow{2}{*}{$\theta$} & \multirow{2}{*}{ Convective terms } \\
\hline$t$ & Time & & \\
\hline$u$ & Velocity & \multirow{2}{*}{$\zeta$} & \multirow{2}{*}{ Non-dimensional time } \\
\hline $\boldsymbol{U}$ & Freestream velocity & & \\
\hline$w_{i}$ & Equilibrium distribution weight & $\psi(I)$ & TVD limiting functions \\
\hline$x, y$ & Coordinate directions & \multicolumn{2}{|c|}{ Subscripts } \\
\hline$\alpha$ & Thermal diffusivity & $G P$ & Ghost Point \\
\hline$\grave{\beta}$ & $\begin{array}{l}\text { The gradient of the general macroscopic } \\
\text { variable }\end{array}$ & $I P$ & Image Point \\
\hline$\beta$ & General macroscopic variables & $B I$ & Boundary intersection \\
\hline$\lambda$ & Period per cycle & \multicolumn{2}{|c|}{ Superscripts } \\
\hline$v$ & Kinematic viscosity & $e q$ & Equilibrium \\
\hline$\rho$ & Density & neq & Nonequilibrium \\
\hline
\end{tabular}

\section{REFERENCES}

[1] W. Shyy, Y. Lian, J. Tang, D. Viieru, H. Liu, Aerodynamics of Low Reynolds Number Flyers, Cambridge University Press, 2007. https://doi.org/10.1017/CB09780511551154

[2] S.P.R. Febi Ponwin, S. Rajkumar, Methods for Improving Lift Force of Wind Turbine Aerofoil Blades during Low Wind Speed Conditions - A Review, Applied Mechanics and Materials, Vol. 787, pp. 134-137, 2015.

https://doi.org/10.4028/www.scientific.net/amm.787.134

[3] N. Bekka, R. Bessaïh, M. Sellam, A. Chpoun, Numerical study of heat transfer around the small scale airfoil using various turbulence models, Numerical Heat Transfer, Part A: Applications, Vol. 56, No. 12, pp. 946-969, 2009.

https://doi.org/10.1080/10407780903508005

[4] S. Succi, Lattice Boltzmann 2038, Europhysics Letters, Vol. 109, No. 5, 2015. https://doi.org/10.1209/0295-5075/109/50001

[5] W. Shyy et al., Recent progress in flapping wing aerodynamics and aeroelasticity, Progress in Aerospace Sciences, Vol. 46, No. 7, pp. 284-327, 2010.

https://doi.org/10.1016/i.paerosci.2010.01.001

[6] B. Dorschner, S.S. Chikatamarla, I.V. Karlin, Transitional flows with the entropic lattice Boltzmann method, Journal of Fluid Mechanics, Vol. 824, pp. 388-412, 2017.

https://doi.org/10.1017/jfm.2017.356

[7] S. Di Francesco, C. Biscarini, P. Manciola, Numerical simulation of water free-surface flows through a front-tracking lattice Boltzmann approach, Journal of Hydroinformatics, Vol. 17, No. 1, pp. 1-6, 2015. https://doi.org/10.2166/hydro.2014.028

[8] D. Chiappini, Numerical simulation of natural convection in open-cells metal foams, International Journal of Heat and Mass Transfer, Vol. 117, 527-537, 2018.

https://doi.org/10.1016/j.ijheatmasstransfer.2017.10.022 
[9] M. Watari, Is the Lattice Boltzmann Method Applicable to Rarefied Gas Flows? Comprehensive Evaluation of the Higher-Order Models, Journal of Fluids Engineering, Transactions of the ASME, Vol. 138, No. 1, 2016. https://doi.org/10.1115/1.4031000

[10] Y. Zhang, A. Xu, G. Zhang, Z. Chen, Discrete Boltzmann method with Maxwell-type boundary condition for slip flow, Communications in Theoretical Physics, Vol. 69, No. 1, pp. 77-85, 2017. https://doi.org/10.1088/0253-6102/69/1/77

[11] Y. Li, C. Liu, X. Liu, Y. Liu, Numerical Simulation of Fluid Flow and Heat Transfer on the Lubricating Surface with Micro-Groove, Heat Transfer-Asian Research, Vol. 45, No. 3, pp. 228-238, 2016. https://doi.org/10.1002/htj.21160

[12] H. Lai, A. Xu, G. Zhang, Y. Gan, Y. Ying, S. Succi, Nonequilibrium theomachy-hydrodynamic effects on the Rayleigh-Taylor instability incompressible flows, Physical Review E, Vol. 94, No. 2, 2016. https://doi.org/10.1103/PhysRevE.94.023106

[13] N. Pellerin, S. Leclaire, M. Reggio, An implementation of the Spalart-Allmaras turbulence model in a multi-domain lattice Boltzmann method for solving turbulent airfoil flows, Computers \& Mathematics with Applications, Vol. 70, No. 12, pp. 3001-3018, 2015.

https://doi.org/10.1016/j.camwa.2015.10.006

[14] J. Tomiyasu, T. Inamuro, Numerical simulations of gas-liquid two-phase flows in a microporous structure, The European Physical Journal Special Topics, Vol. 171, pp. 123127, 2009. https://doi.org/10.1140/epjst/e2009-01019-5

[15] R. Matin, M.K. Misztal, A. Hernández-García, J. Mathiesen, Evaluation of the finite element lattice Boltzmann method for binary fluid flows, Computers \& Mathematics with Applications, Vol. 74, No. 2, pp. 281-291, 2017.

https://doi.org/10.1016/j.camwa.2017.04.027

[16] C.T. Orlowski, A.R. Girard, Modeling and Simulation of Nonlinear Dynamics of Flapping Wing Micro Air Vehicles, AIAA Journal, Vol. 49, No. 5, pp. 969-981, 2011.

https://doi.org/10.2514/1.J050649

[17] A. Tiwari, S.P. Vanka, A ghost fluid Lattice Boltzmann method for complex geometries, International Journal for Numerical Methods in Fluids, Vol. 69, No. 2, pp. 481-498, 2012.

https://doi.org/10.1002/fld.2573

[18] J.M. Anderson, K. Streitlien, D.S. Barrett, M.S. Triantafillou, Oscillating foils of high propulsive efficiency, Journal of Fluid Mechanics, Vol. 360, pp. 41-72, 1998.

https://doi.org/10.1017/S0022112097008392

[19] Z. Guo, C. Zheng, B. Shi, An extrapolation method for boundary conditions in lattice Boltzmann method, Physics of Fluids, Vol. 14, No. 6, 2002.

https://doi.org/10.1063/1.1471914

[20] C. Hirsch, Numerical Computation of Internal and External Flows: The Fundamentals of Computational Fluid Dynamics, Oxford: Butterworth-Heinemann, 2007.

[21] H.K. Versteeg and W. Malalasekera, An Introduction to Computational Fluid Dynamics: The Finite Volume Method, New York: Pearson Education, 2007.

[22] P.L. Roe, Some contributions to the modelling of discontinuous flows, in: Large-scale computations in fluid mechanics; Proceedings of the $15^{\text {th }}$ Summer Seminar on Applied 
Mathematics, Part 2, La Jolla, CA, 1983. Providence, RI, American Mathematical Society, pp. 163-193, 1985. https://ui.adsabs.harvard.edu/abs/1985ams..conf..163R

[23] M.B. Bragg, An experimental study of a NACA0012 airfoil aerodynamics with a simulated glaze ice accretion, Vol. 2, 1993. https://ntrs.nasa.gov/search.jsp?R=19930013634

[24] Y. Lian, W. Shyy, Aerodynamics of Low Reynolds Number Plunging Airfoil Under Gusty Environment, in 45th AIAA Aerospace Sciences Meeting and Exhibit, American Institute of Aeronautics and Astronautics, Reston, Virigina, 2007.

https://doi.org/10.2514/6.2007-71

[25] D.F. Hinz, H. Alighanbari, C. Breitsamter, Influence of heat transfer on the aerodynamic performance of a plunging and pitching NACA0012 airfoil at low Reynolds numbers, Journal of Fluids and Structures, Vol. 37, pp. 88-99, 2013.

https://doi.org/10.1016/j.jfluidstructs.2012.08.012

[26] F.P. Incropera, D.P. DeWitt, Introduction to Heat Transfer, 4 ${ }^{\text {th }}$ Edition, Wiley, 2001.

[27] D.J. Norton, J.M. Macha, J.C. Young, Surface temperature effect on subsonic stall, Journal of Spacecraft and Rockets, Vol. 10, No. 9, pp. 581-587, 1973.

https://doi.org/10.2514/3.61929 